\title{
Skeletal muscle fat oxidation: timing and flexibility are everything
}

\author{
David E. Kelley
}

Obesity and Nutrition Research Center, University of Pittsburgh School of Medicine, Pittsburgh, Pennsylvania, USA.

\begin{abstract}
In order to examine the factors governing the timing and flexibility of skeletal muscle switching between fat and carbohydrate oxidation, Ukropcova et al. studied the effect of glucose and fatty acid availability on the preference for fat oxidation in myocytes cultured from human male quadriceps muscle taken from subjects with varied BMI, fat mass, and insulin sensitivity (see the related article beginning on page 1934). The authors found that in vivo insulin sensitivity was related to a higher in vitro capacity for fat oxidation. These findings support the concept that the capacity of skeletal muscle to oxidize fat under appropriate physiological conditions is related to leanness, aerobic fitness, and insulin sensitivity.
\end{abstract}

\section{Muscle's transition from glucose to fat oxidation}

Skeletal muscle adapts to 2 quite opposite physiological conditions - reduced energy intake during fasting and increased energy expenditure during sustained exercise - by increasing its reliance upon fat oxidation (1). The transition to higher reliance upon fat oxidation spares utilization of plasma glucose during fasting and delays consumption of muscle glycogen during exercise. These are well-recognized homeostatic adaptations. Indeed, nearly 50 years ago, Andres, Cader, and Zierler reported in the JCI that even in lean, metabolically healthy volunteers after just an overnight fast, skeletal muscle chiefly relies upon fat oxidation (2). More recently, it has been proposed that inability to increase reliance upon fat oxidation is related to the pathogenesis of insulin resistance in skeletal muscle and perhaps to the pathogenesis of obesity $(3,4)$. In this issue of the JCI, using a muscle culture system prepared from biopsy of human vastus lateralis, Ukropcova et al. (5) examined the capacity for fat oxidation by skeletal muscle and found that it was increased in subjects with increased insulin sensitivity, leanness, and aerobic fitness (Figure 1).

Nonstandard abbreviations used: DM, diabetes mellitus; RQ, respiratory quotient.

Conflict of interest: The author has declared that no conflict of interest exists.

Citation for this article: J. Clin. Invest. 115:1699-1702 (2005). doi:10.1172/JCI25758.
Fatty acids induce insulin resistance Skeletal muscle has a key role in determining systemic insulin sensitivity because under insulin-stimulated conditions, a major proportion of glucose utilization occurs in muscle. Impaired glucose utilization in muscle determines the severity of systemic insulin resistance in such common metabolic diseases as type 2 diabetes mellitus (DM) and obesity. In order to evaluate whether muscle's impaired capacity to increase reliance upon fat oxidation is related to the pathogenesis of insulin resistance, one must first address the apparently contradictory observation that insulin resistance in muscle can be induced by elevated plasma fatty acids levels $(6,7)$ and that such elevations are associated with increased fat oxidation. Even experimental maintenance of fasting levels of plasma fatty acids, which prevent the suppression that normally occurs during elevation of insulin, is sufficient to significantly lower insulin-stimulated glucose uptake by skeletal muscle, impair insulin suppression of lipid oxidation, and blunt stimulation of glucose oxidation in muscle (8). These experimental conditions, which induce insulin resistance in skeletal muscle by elevating plasma fatty acids, have a strong congruence with traits found in obesity and type $2 \mathrm{DM}(9,10)$. The strength and consistency of such observations make it challenging to reconcile them with the notion that insulin-resistant skeletal muscle can also manifest reduced efficiency of fat oxidation during fasting metabolism despite elevated levels of plasma FFA as is usually present in obesity and type 2 DM.
Fat oxidation by muscle as a component of insulin sensitivity

The effects of aerobic exercise on muscle physiology and muscle's capacity for fat oxidation illustrate that insulin-sensitive skeletal muscle manifests a robust reliance on fat oxidation during postabsorptive metabolism, yet it also manifests keen reliance on glucose oxidation during insulin-stimulated conditions. One aspect of the training responses to aerobic exercise is increased reliance upon fat oxidation during physical activity. Training also enhances muscle sensitivity to insulin-mediated glucose uptake. Therefore, an increase in capacity for fat oxidation by muscle can occur concurrently with an increase in the capacity for insulin-mediated glucose uptake and metabolism. Also, while a range of methods (histology, bioimaging, and lipid extraction) have shown an association between insulin resistance and increased intramyocellular lipid content (11), in lean, insulin-sensitive, endurance-trained athletes, muscle lipid content is relatively high (12). It seems likely that in endurance-trained athletes, a high content of muscle triglyceride provides a reservoir for high rates of oxidation, and that the pool of intramyocellular triglyceride undergoes exercise-induced depletion and postprandial repletion.

The question therefore arises: does the accretion of muscle triglyceride represent different kinetics for sedentary as opposed to overweight individuals, namely, slower fractional turnover of triglycerides and a relative impairment in fat oxidation? Measurement of the respiratory quotient (RQ; the quotient of $\mathrm{CO}_{2}$ production and $\mathrm{O}_{2}$ consumption, which reflects the macronutrient mix being oxidized) across the tissue bed of the leg in lean, metabolically healthy volunteers reveals a high reliance on fat oxidation (lower RQ) during fasting conditions (Figure 1A). In these individuals, insulin infusion readily suppresses this preference for fat oxidation as muscle shifts to high reliance upon glucose oxidation (higher RQ) (13) (Figure 1C). Thus, metabolically 


\section{A Metabolically flexible}

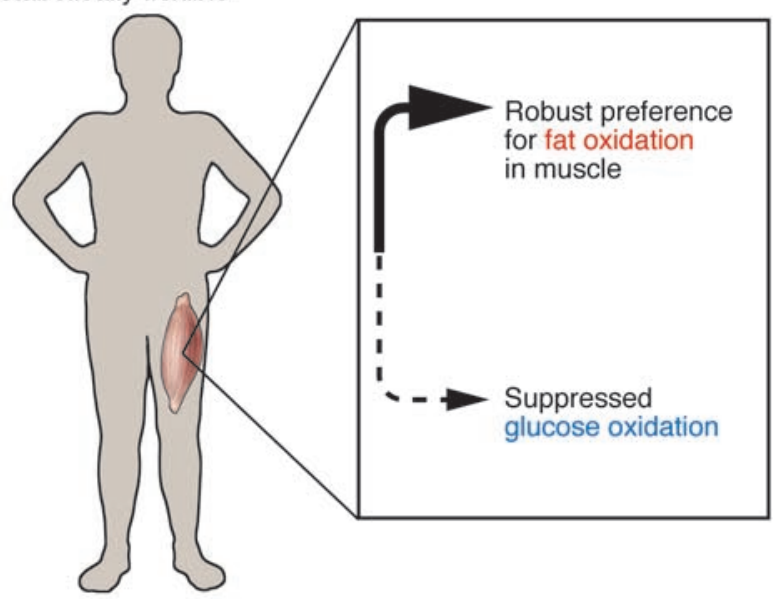

Lean, aerobically fit individual
B Metabolically inflexible

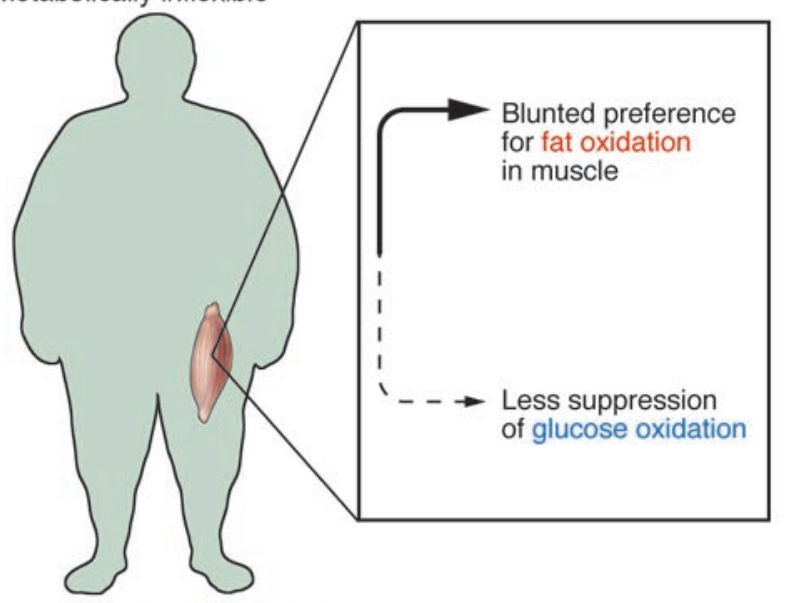

Obese, aerobically unfit individual

During insulin-stimulated conditions

\section{Metabolically flexible}

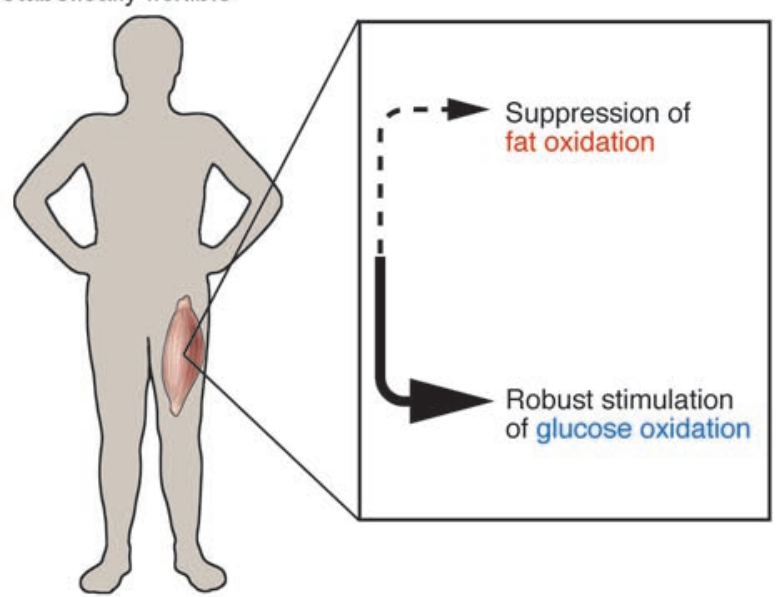

D Metabolically inflexible

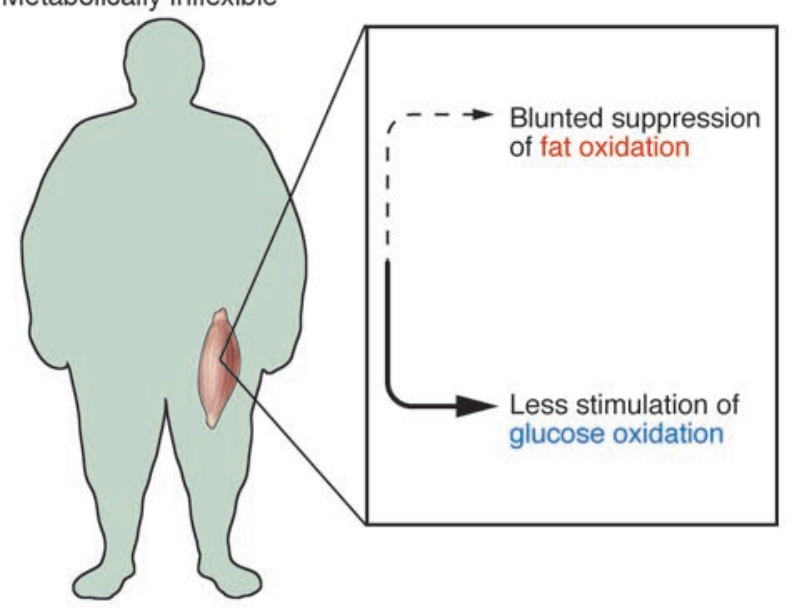

Figure 1

The flexibility of skeletal muscle to switch between oxidizing fat and glucose is related to insulin sensitivity, percentage of body fat, and fitness. Differences in the patterns of glucose and fat oxidation in skeletal muscle during fasting conditions are shown in a lean, aerobically fit individual (A), in whom there is a high reliance upon fat oxidation during fasting conditions, and in an obese, sedentary individual (B), in whom there is less reliance on fat and a greater reliance on glucose oxidation. The patterns of glucose and fat oxidation during insulin-stimulated conditions are also shown. In skeletal muscle of a lean, aerobically fit individual (C), insulin strongly suppresses fat oxidation and induces a high reliance upon glucose oxidation, whereas in skeletal muscle of an obese, sedentary individual (D), there is less stimulation of glucose oxidation by insulin and blunted suppression of fat oxidation. Thus, in skeletal muscle of obese, sedentary individuals, there is a constricted range in switching between fat and glucose oxidation compared to the dynamic switching evident in aerobically fit, lean individuals. This constrained homeostatic adjustment to the transitions between fasting and insulin-stimulated conditions in obese, sedentary individuals has been described as "metabolic inflexibility" of skeletal muscle.

healthy skeletal muscle is characterized by the ability to switch easily between glucose and fat oxidation in response to homeostatic signals. The skeletal muscle in individuals with type $2 \mathrm{DM}$ and obesity shows a great reduction in this metabolic flexibility. RQ values measured across the tissue bed of the leg during fasting are elevated in type $2 \mathrm{DM}$ and in obesity (14-16), and yet stimulation of glucose oxidation in response to insulin is blunted. This flat or unresponsive pattern, responding inefficiently both to the stimulus of fasting to enhance fat oxidation (Figure 1B) and to the stimulus of insulin to enhance glucose oxidation (Figure 1D), has been described as "metabolic inflexibility." It is this physiological phenotype that Ukropcova and colleagues have explored using cultured myocytes (5). A prior study found that a diminished reliance on fat oxidation by skeletal muscle during fasting conditions is predictive of weight gain (17). A program of moderate weight loss and improved aerobic fitness restores metabolic flexibility in overweight and obese insulin-resistant men and women, and this improvement in metabolic flexibility 
correlated strongly with improvement in insulin-stimulated glucose disposal (18). Interestingly, however, moderate weight loss achieved without a change in aerobic fitness does not appear to improve capacity for fat oxidation, yet does improve suppression of fat oxidation during insulin-stimulated conditions (19-21).

\section{Susceptibility to inhibition of fat oxidation}

Metabolic flexibility has 2 facets: insulin suppresses fat oxidation and stimulates glucose oxidation, while fasting stimulates a reliance on fat oxidation. Earlier, the ability of elevated plasma FFA levels to impede insulin-stimulated glucose oxidation was discussed. In the study by Ukropcova and colleagues (5), a finding of particular interest was the variable effect of glucose on the suppression of fat oxidation in myocytes cultured from the quadriceps of healthy males. Resistance to suppression of fat oxidation by hyperglycemia was found to be related to leanness, aerobic fitness, and insulin sensitivity. These in vitro findings resonate with the results of earlier clinical investigations showing that experimental induction of hyperglycemia while maintaining low circulating insulin levels (via somatostatin suppression of insulin secretion) induced elevated RQ values in the leg tissue of volunteers, a response that was more pronounced in obese than in lean individuals $(22,23)$.

\section{Potential cellular mechanisms for metabolic flexibility in fat oxidation}

A prior study found that characteristics of muscle insulin resistance are retained in myocyte culture (24), and it has been reported that muscle obtained from patients with type $2 \mathrm{DM}$ has a reduced capacity for fat oxidation in culture (25). The results reported by Ukropcova et al. (5) provide the additional insight that properties of metabolic flexibility in the transition between fat and glucose oxidation that are manifest in vivo are retained in myocyte cultures. What are the cellular characteristics of myocytes that determine metabolic flexibility? The study by Ukropcova et al. serves as an impetus for further research as the answers to these questions are presently unclear, but mitochondria may play a critical role. Competition between glucose and fat oxidation can occur at several locations in the mitochondria. One juncture is the mitochondrial outer membrane between the relative activities of pyruvate dehydrogenase and carnitine palmitoyl transferase complexes. Perhaps this competition also occurs within the matrix of the mitochondria in interactions between the $\beta$-oxidation and TCA cycles and in delivering $\mathrm{FADH}_{2}$ and $\mathrm{NADH}$ to different loci within the electron transport chain, which is embedded within the inner mitochondrial membrane. During the past several years, interest in the role that mitochondria might play in the pathogenesis of muscle insulin resistance has rapidly grown (2632). In addition to clinical investigations, suitable animal models for further study are also being developed. For example, rats selectively bred for low oxidative enzyme activity in skeletal muscle develop a metabolic syndrome phenotype (33). In clinical studies examining the physiological phenotype of metabolic flexibility, muscle biopsy samples have shown reduced oxidative enzyme activity (34-36), and improvement in metabolic inflexibility following exercise training was found to cause improvement in mitochondrial metabolism (37). With regard to the experimental use of muscle cultures, it will be of great interest to more fully examine mitochondrial metabolism as well as to undertake broader analyses of known metabolic pathways in order to elucidate the mechanisms that permit or constrain the metabolic adaptation of skeletal muscle toward a high rate of fat oxidation.

Address correspondence to: David E. Kelley, 807N Montefiore-University of Pittsburgh Medical Center, 3459 Fifth Avenue, Pittsburgh, Pennsylvania 15213, USA. Phone: (412) 692-2848; Fax: (412) 692-2165; E-mail: Kelley@dom.pitt.edu.

1. Henriksson, J. 1995. Muscle fuel selection: effect of exercise and training [review]. Proc. Nutr. Soc. 54:125-138.

2. Andres, R., Cader, G., and Zierler, K. 1956. The quantitatively minor role of carbohydrate in oxidative metabolism by skeletal muscle in intact man in the basal state. Measurement of oxygen and glucose uptake and carbon dioxide and lactate production in the forearm. J. Clin. Invest. 35:671-682.

3. Kelley, D., and Mandarino, L. 2000. Fuel selection in human skeletal muscle in insulin resistance: a reexamination [review]. Diabetes. 49:677-683.

4. Storlien, L., Oakes, N., and Kelley, D. 2004. Metabolic flexibility. Proc. Nutr. Soc. 63:363-368.

5. Ukropcova, B., et al. 2005. Dynamic changes in fat oxidation in human primary myocytes mirror metabolic characteristics of the donor. J. Clin. Invest. 115:1934-1941. doi:10.1172/JCI24332.

6. Boden, G., et al. 1994. Mechanisms of fatty acidinduced inhibition of glucose uptake. J. Clin. Invest. 93:2438-2446.

7. Boden, G., et al. 2001. Effects of acute changes of plasma free fatty acids on intramyocellular fat content and insulin resistance in healthy subjects. Diabetes. 50:1612-1617.
8. Kelley, D., et al. 1993. Interaction between glucose and free fatty acid metabolism in human skeletal muscle. J. Clin. Invest. 92:93-98.

9. Lillioja, S., et al. 1985. Relationship between insulin-mediated glucose disposal and lipid metabolism in man. J. Clin. Invest. 75:1106-1115.

10. DeFronzo, R. 1988. Lilly lecture 1987. The triumvirate: beta-cell, muscle, liver. A collusion responsible for NIDDM [review]. Diabetes. 37:667-687.

11. Perseghin, G., et al. 1999. Intramyocellular triglyceride content is a determinant of in vivo insulin resistance in humans: a $1 \mathrm{H}-13 \mathrm{C}$ nuclear magnetic resonance spectroscopy assessment in offspring of type 2 diabetic parents. Diabetes. 48:1600-1606.

12. Goodpaster, B., et al., 2001. Skeletal muscle lipid content and insulin resistance: evidence for a paradox in endurance-trained athletes. J. Clin. Endocrinol. Metab. 86:5755-5761.

13. Kelley, D., et al. 1990. Effects of insulin on skeletal muscle glucose storage, oxidation, and glycolysis in man. Am. J. Physiol. Endocrinol. Metab. 258:E923-E929.

14. Colberg, S.R., et al. 1995. Skeletal muscle utilization of free fatty acids in women with visceral obesity. J. Clin. Invest. 95:1846-1853.

15. Kelley, D., Mokan, M., and Veneman, T. 1994. Impaired postprandial glucose utilization in noninsulin-dependent diabetes mellitus. Metabolism. 43:1549-1557.

16. Kelley, D.E., et al. 1999. Skeletal muscle fatty acid metabolism in association with insulin resistance, obesity, and weight loss. Am. J. Physiol. 277:E1130-E1141.

17. Zurlo, F., et al. 1990. Low ratio of fat to carbohydrate oxidation as predictor of weight gain: A study of 24-h RQ. Am. J. Physiol. 259:E650-E657.

18. Goodpaster, B.H., Katsiaras, A., and Kelley, D.E. 2003. Enhanced fat oxidation through physical activity is associated with improvements in insulin sensitivity in obesity. Diabetes. 52:2191-2197.

19. Simoneau, J.A., et al. 1999. Markers of capacity to utilize fatty acids in human skeletal muscle: relation to insulin resistance and obesity and effects of weight loss. FASEB J. 13:2051-2060.

20. Raben, A., Mygind, E., and Astrup, A. 1998. Lower activity of oxidative key enzymes and smaller fiber areas in skeletal muscle of postobese women. Am.J. Physiol. 1998. 275:E487-E494.

21. Astrup, A., et al. 1994. Failure to increase lipid oxidation in response to increasing dietary fat content in formerly obese women. Am. J. Physiol. 266:E592-E599.

22. Mandarino, L.J., et al. 1996. Interaction of carbohydrate and fat fuels in human skeletal muscle: impact of obesity and NIDDM. Am. J. Physiol. 270:E463-E470.

23. Sidossis, L., et al. 1996. Glucose plus insulin regulate fat oxidation by controlling the rate of fatty acid entry into the mitochondria. J. Clin. Invest. 98:2244-2250.

24. Henry, R.R., et al. 1995. Insulin action and glucose metabolism in nondiabetic control and NIDDM subjects. Comparison using human skeletal muscle cell cultures. Diabetes. 44:936-946.

25. Gaster, M., et al. 2004. Reduced lipid oxidation in skeletal muscle from type 2 diabetic subjects may be of genetic origin: evidence from cultured myotubes. Diabetes. 53:542-548.

26. Petersen, K., et al. 2003. Mitochondrial dysfunction in the elderly: possible role in insulin resistance. Science. 300:1140-1142.

27. Petersen, K., et al. 2004. Impaired mitochondrial activity in the insulin-resistant offspring of patients with type 2 diabetes. N. Engl. J. Med. 350:664-671.

28. Patti, M., et al. 2003. Coordinated reduction in genes of oxidative metabolism in humans with insulin resistance and diabetes: potential roles of PGC1 and NRF-1. Proc. Natl. Acad. Sci. U. S. A. 
100:8466-8471.

29. Mootha, V., et al. 2003. PGC-1alpha responsive genes involved in oxidative phosphorylation are coordinately downregulated in human diabetes. Nat. Genet. 34:267-273.

30. Stump, C., et al. 2003. Effect of insulin on human skeletal muscle mitochondrial ATP production, protein synthesis and mRNA transcripts. Proc. Natl. Acad. Sci. U. S. A. 100:7996-8001.

31. Kelley, D.E., et al. 2002. Dysfunction of mitochondria in human skeletal muscle in type 2 diabetes.
Diabetes. 51:2944-2950.

32. Ritov, V., et al. 2005. Deficiency of sub-sarcolemmal mitochondria in obesity and type 2 diabetes. Diabetes. 55:8-14.

33. Wisloff, U., et al. 2005. Cardiovascular risk factors emerge after artificial selection for low aerobic capacity. Science. 307:418-420.

34. Simoneau, J., and Kelley, D. 1997. Altered glycolytic and oxidative capacities of skeletal muscle contribute to insulin resistance in NIDDM. J. Appl. Physiol. 83:166-171.
35. Simoneau, J.-A., et al. 1999. Markers of capacity to utilize fatty acids in human skeletal muscle: relation to insulin resistance and obesity and effects of weight loss. FASEB J. 13:2051-2060.

36. Zurlo, F., et al. 1994. Whole body energy metabolism and skeletal muscle biochemical characteristics. Metabolism. 43:481-486.

37. Menshikova, E., et al. 2005. Effects of weight loss and physical activity on skeletal muscle mitochondrial function in obesity. Am. J. Physiol. Endocrinol. Metab. 288:E818-E825.

\title{
HIF-1 $\alpha$ : a master regulator of innate host defenses?
}

\author{
Kol A. Zarember and Harry L. Malech
}

Laboratory of Host Defenses, National Institute of Allergy and Infectious Diseases, National Institutes of Health, Bethesda, Maryland, USA.

In the days following infection, when the human body develops and refines antibodies and prepares to mount an adaptive immune response, the bulwark of innate host defense against microbial infection is the polymorphonuclear leukocyte (PMN). PMNs seek out, identify, engulf, and sterilize invading microbes using both $\mathrm{O}_{2}$-dependent and $\mathrm{O}_{2}$-independent antimicrobial systems. A decrease in PMN numbers or function caused by immunosuppression or disease increases the risk of infection. In this issue of the JCI, Peyssonnaux et al. identify a novel and essential role for hypoxia-inducible factor- $1 \alpha$ in regulating several important PMN functions relevant to host defense, including transcription of cationic antimicrobial polypeptides and induction of NO synthase (see the related article beginning on page 1806).

Hypoxia-inducible factor 1 (HIF-1) is a multisubunit protein that regulates transcription at hypoxia response elements (HREs) and is composed of 2 basic helix-loop-helix proteins: the $\alpha$ subunit, HIF- $1 \alpha$, and the constitutively expressed HIF- $1 \beta$ (also known as aryl hydrocarbon receptor nuclear translocator [ARNT]). As depicted in Figure 1A, during normoxia HIF- $1 \alpha$ is hydroxylated on several proline and asparaginyl residues, which enables high-affinity binding of HIF- $1 \alpha$ to von Hippel-Lindau tumor suppressor protein (vHL), a component of a ubiquitin ligase complex that ubiquitinates and thereby targets HIF- $1 \alpha$ for proteosomal degradation. Under hypoxic conditions the

Nonstandard abbreviations used: CEBP, CCAAT enhancer-binding protein; CRAMP, cathelicidin-related antimicrobial peptide; FIH, factor inhibiting HIF-1 $\alpha$; HIF-1, hypoxia-inducible factor 1 ; HRE, hypoxic response element; PMN, polymorphonuclear leukocyte; vHL, von Hippel-Lindau tumor suppressor protein.

Conflict of interest: The authors have declared that no conflict of interest exists.

Citation for this article: J. Clin. Invest. 115:1702-1704 (2005). doi:10.1172/JCI25740.
$\mathrm{O}_{2}$-dependent hydroxylation of HIF- $1 \alpha$ is decreased, which prevents its degradation (Figure 1B). A further level of $\mathrm{O}_{2}$-dependent regulation exists: the hydroxylation of an asparagine residue by factor inhibiting HIF-1 $\alpha$ (FIH) blocks the interaction of HIF- $1 \alpha$ with p300/CBP transcriptional coactivator proteins, thereby decreasing transcription of HIF- $1 \alpha$-regulated genes at normoxia. When HIF-1 $\alpha$ levels increase in response to hypoxia in tissues (or after stimulation of myeloid cells with bacteria, as discussed below), functional HIF-1 regulates transcription at HREs of target gene regulatory sequences, which results in the transcription of genes such as Erythropoietin and VEGF and thereby enhances local vascularization and systemic oxygen transport (Figure 1B). The details of the regulation of the general hypoxia response have been thoroughly discussed in several reviews (1-3).

\section{A role for HIF-1 $\alpha$ in myeloid cells}

The knockout of HIF- $1 \alpha$ in mice is embryonically lethal due to its essential role in the development of the vascula- ture. To circumvent this lethality and allow further examination of the role of HIF- $1 \alpha$ in phagocytes, Cramer et al. employed a myeloid cell-specific HIF- $1 \alpha-$ knockout approach and found that HIF- $1 \alpha$ was an important regulator of myeloid cell metabolism (by decreasing cellular ATP levels), neutrophil bactericidal potency, and macrophage migration (4). In this issue of the JCI, Peyssonnaux et al. (5) built on this foundation and attempted to understand the specific molecular defects contributing to depressed myeloid cell function in the absence of HIF- $1 \alpha$. Temporarily leaving aside the consequences of the absence of HIF-1 $\alpha$, the authors also discovered that in addition to being activated by hypoxia, HIF-1 $\alpha$ was equivalently upregulated in WT macrophages at normoxia following exposure to Gram-positive (Streptococcus and Staphylococcus) and Gram-negative (Salmonella and Pseudomonas) bacteria. HIF-1 $\alpha$ was previously shown to be activated in macrophages treated with LPS (6), a microbial activator of TLR-4. The 10 human TLRs are the cellular sentinels of microbial recognition. They respond to a variety of microbial products (e.g., LPS, lipoproteins, proteins, and nucleic acids) by activating signaling pathways leading to NF-кB-mediated transcriptional regulation and, in some cases, activation of Rac1 and PI3K, which may regulate more rapid cellular responses (7). Regardless of the exact position along their signal cascades at which the TLR and HIF-1 $\alpha$ pathways intersect, these observations illuminate a fertile territory for further study. To what extent, if any, do the transcrip- 